\title{
DETECTING NEGATIVE CURVATURE IN GROUPS VIA LOCAL CONDITIONS
}

\author{
PAUL SHAWCROFT
}

(Communicated by James West)

\begin{abstract}
We characterize negative curvature of groups in terms of properties which can be checked locally in the Cayley graph. We prove the equivalence of these properties with the more familiar thin triangles property. We also present an algorithm which, when given a finite presentation of a group $G$, will recognize these properties in finite time if $G$ is negatively curved.
\end{abstract}

\section{INTRODUCTION}

In this paper we present a new characterization of negatively curved or Gromov-hyperbolic groups. We prove that this new characterization is equivalent to the more familiar definition which is stated in terms of thin triangles. We also show that this new characterization leads to a partial algorithm for verifying negative curvature in groups.

The distinctive feature of our new characterization is that we can verify it by looking at neighborhoods of finitely many vertices of the Cayley graph which arises from some finite presentation. It is known that every negatively curved group admits a finite presentation, thus we restrict ourselves to that category. See [Gr, 1.7 and 2.2], [GdlH, Proposition 4.17]. We refer to the components of our new characterization as Local Conditions.

0.1. Setting. Let $G=\langle A \mid R\rangle$ be a group with a fixed finite presentation. Let $\Gamma$ be the Cayley graph of $G$ with respect to this presentation. We consider each edge of $\Gamma$ to be the isometric image of $[0,1]$, and thus $(\Gamma, d)$ is a connected geodesic metric space: given any two points there exists a path joining them which realizes the distance between them. We will let $[x, y]$ denote a (possibly nonunique) geodesic segment joining the points $x, y \in \Gamma$.

We say that $G$ is negatively curved or Gromov-hyperbolic if geodesic triangles in $\Gamma$ are uniformly thin: there exists a number $\delta \geq 0$ such that given any geodesic triangle, a point on one side is within $\delta$ of some point in the union of the other two sides. We refer to this condition by saying that $G$ has thin triangles $(\delta)$; if there is no need to specify $\delta$, we say simply that $G$ has thin triangles. We may take $\delta$ to be a nonnegative integer.

Received by the editors March 31, 1993.

1991 Mathematics Subject Classification. Primary 20E25, 20E34, 20F05, 20F10.

Thanks to The Geometry Center for providing the computers used during the research. Thanks also to the BYU Mathematics Department and James Cannon. 
Negative curvature is a property of the group and is independent of presentation. If two distinct finite presentations give rise to isomorphic groups, then the corresponding Cayley graphs both have thin triangles, though possibly with different values of $\delta$ in each. See [GdlH, Corollary 5.14], [Ge], [Gh]. For more discussion on negatively curved groups see $[\mathrm{B}],[\mathrm{C}],[\mathrm{ABC}]$.

For any path $P$ in $\Gamma$ let $\ell(P)$ denote the length of $P$. Let $O$ denote a fixed vertex of $\Gamma$, and for $n \geq 0$, let $B(n), R(n)$, and $S(n)$ denote the closed ball, open ball, and sphere of radius $n$ centered at $O$. Let $A[m, n]$ denote the annulus of inner radius $m$ and outer radius $n$. For an arbitrary vertex $y$, let $B(y, n)$ denote the ball of radius $n$, or $n$-ball, centered at $y$. Precise definitions are as follows:

$$
\begin{gathered}
B(n)=\{x \in \Gamma \mid d(O, x) \leq n\}, \\
R(n)=\{x \in \Gamma \mid d(O, x)<n\}, \\
S(n)=\{x \in \Gamma \mid d(O, x)=n\}, \\
A[m, n]=\{x \in \Gamma \mid m \leq d(O, x) \leq n\}, \\
B(y, n)=\{x \in \Gamma \mid d(x, y) \leq n\} .
\end{gathered}
$$

\section{Conditions}

1.1. Definitions and conditions. We begin by defining radial geodesic and radial quasi-geodesic.

Definition 1.1. An edge path $V \subset \Gamma$ is a radial geodesic if for any edge path $P \subset V$ with endpoints $p_{1}$ and $p_{2}, \ell(P)=\left|d\left(O, p_{1}\right)-d\left(O, p_{2}\right)\right|$.

Definition 1.2. Let $j$ and $j^{\prime}$ be positive integers. An edge path $U$ is a radial $\left(j, j^{\prime}\right)$-quasi-geodesic if for any edge path $P \subset U$ with endpoints $p_{1}$ and $p_{2}$, if $\ell(P) \geq j$, then $\ell(P) \leq j^{\prime}\left|d\left(O, p_{1}\right)-d\left(O, p_{2}\right)\right|$.

Remark 1.3. A radial geodesic is a radial $(j, 1)$-quasi-geodesic for any $j$. A radial quasi-geodesic varies only slightly from a true radial geodesic. The variable $j^{\prime}$ gives a bound on the variation, while $j$ tells us how long of a subsegment we need in order to detect this variation.

Remark 1.4. If $U$ is a radial $\left(j, j^{\prime}\right)$-quasi-geodesic with endpoints $u_{1}$ and $u_{2}$ and if $\ell(U) \geq j$, then it follows from Definition 2 that $\left|d\left(O, u_{1}\right)-d\left(O, u_{2}\right)\right| \geq$ $\frac{j}{j^{\prime}}$. In particular, $d\left(O, u_{1}\right) \neq d\left(O, u_{2}\right)$. If $d\left(O, u_{1}\right)>d\left(O, u_{2}\right)$, then we say that $u_{1}$ is the outer endpoint of $U$.

We will show that the following conditions are equivalent to thin triangles.

The local conditions. There exist integers $k \geq 2$ and $l \geq 2 k$ such that the following hold. (See Figures 1.1(a)-(c).)

(1) Let $P$ be an edge path in $A[n, n+k]$ for some $n$. If $P$ has one endpoint in $S(n)$ and the other in $S(n) \cup S(n+1)$ and if $P$ leaves $B(n+k-1)$, then there exists an edge path $P^{\prime}$ with the same endpoints as $P$ and $\ell\left(P^{\prime}\right)<\ell(P)$.

(2) Let $P$ be an edge path in $A[n, n+k]$ for some integer $n \geq 0$. If $\ell(P)=l+1$, then there exists an edge path $P^{\prime}$ with the same endpoints as $P$ such that $\ell\left(P^{\prime}\right)<\ell(P)$.

(3) Let $U$ be a geodesic edge path with endpoints $x$ and $z$ such that $d(O, x)-d(O, z)=4 k$. Let $V=[O, y]$ with outer endpoint $y$. Assume 


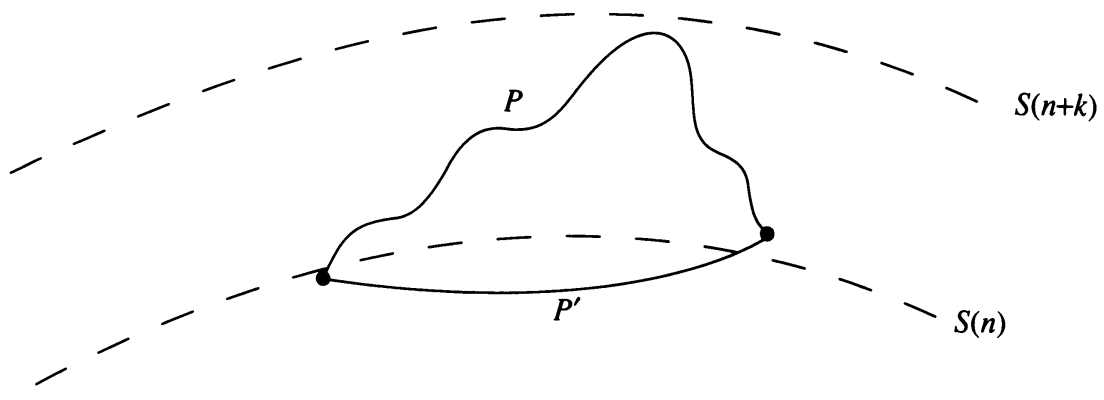

FIGURE 1.1(a)

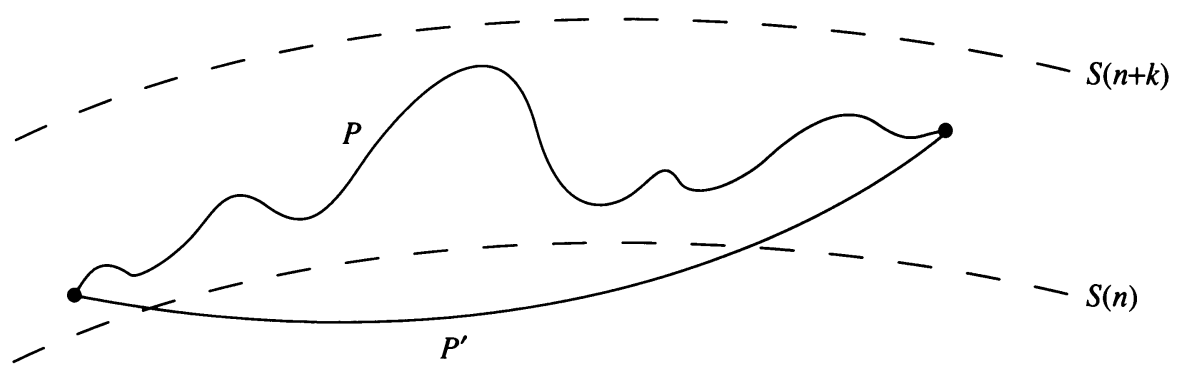

Figure 1.1(b)

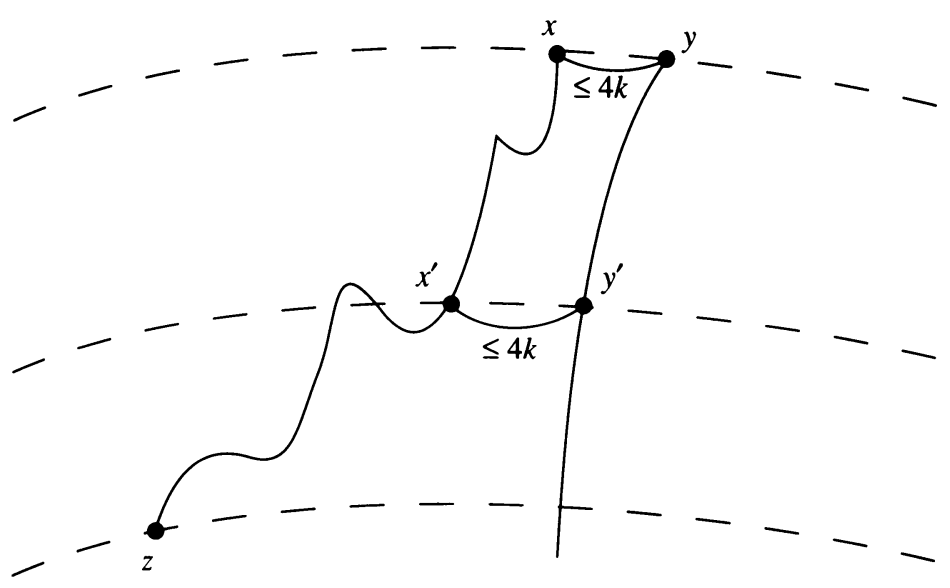

FIGURE 1.1(c)

$d(O, x)=d(O, y)$. Order the points of $U$ from $z$ to $x$ and let $x^{\prime}$ be the maximal vertex of $U \cap S(d(O, x)-2 k)$. Let $y^{\prime}$ be the unique vertex in $V \cap S(d(O, y)-2 k)$. If $d(x, y) \leq 4 k$, then $d\left(x^{\prime}, y^{\prime}\right) \leq 4 k$.

1.2. Verifying the conditions. Assume the local conditions hold in $\Gamma$. We outline a way to verify them. First, we make precise what it means to verify each condition at a vertex $y$.

To check condition (1) at $y$ we look at all edge paths of length at most $l$ 
that have $y$ as an endpoint and that reach out and back in the way described in condition (1), verifying that such paths are not geodesics.

To check condition (2) we look at all edge paths of length $l+1$ having one endpoint at $y$ and lying in some annulus of width $k$, verifying that such paths are not geodesics.

We verify condition (3) at $y$ as follows. Let $x$ be a fixed vertex on the same level as $y$ and such that $d(x, y) \leq 4 k$. Let $U=[z, x]$ be a geodesic with $d(O, x)-d(O, z)=4 k$ and with the points ordered from $z$ to $x$. Let $z^{\prime}$ be the maximal vertex of $U \cap S(d(O, z))$. An application of Theorem 2.1, given below, shows that $\ell\left(\left[z^{\prime}, x\right]\right) \leq 4 k l$. That subsegment is the only part of $U$ we need, so we may as well assume that $\ell(U) \leq 4 k l$. Let $V$ be a radial geodesic segment of length at least $2 k$ with outer endpoint $y$. We verify condition (3) on this $U$ and $V$.

Now we let $x$ and $z$ vary over all vertices such that $d(O, y)=d(O, x)$, $d(O, x)-d(O, z)=4 k$, and $d(x, z) \leq 4 k l$. We also let $V$ vary over other radial geodesic segments with outer endpoint $y$.

We now see the local nature of these conditions. A neighborhood of radius $4 k+4 k l$ contains everything we need to see in order to verify the conditions. Therefore, we set $N=4 k+4 k l$ and we classify the vertices of $\Gamma$ according to their $N$-types. This classification is made precise as follows. Given two vertices $x, y \in \Gamma$ we say that $x$ and $y$ are equivalent (have the same $N$-type) if the graph automorphism $T: \Gamma \rightarrow \Gamma$ taking $x$ to $y$ by left multiplication by $y x^{-1}$ has the property that

$$
d\left(O, x^{\prime}\right)-d(O, x)=d\left(O, T x^{\prime}\right)-d(O, y)
$$

for each $x^{\prime} \in B(x, N)$. In simpler terms, $x \sim y$ if the restricted map $T \mid B(x, N): B(x, N) \rightarrow B(y, N)$ preserves distances from the origin, relative to $x$ and $y$.

Since $G$ is finitely presented, there are only finitely many equivalence classes ( $N$-types) of vertices. To verify the local conditions in $\Gamma$, we choose a vertex from each equivalence class and check the conditions at those vertices, as described above.

\section{CONDITIONS IMPLY NEGATIVE CURVATURE}

In this section we prove that if the local conditions hold in $\Gamma$, then $\Gamma$ has thin triangles. Let $O$ denote a fixed vertex in $\Gamma$.

Conditions (1) and (2) have consequences for the structure of long geodesic segments in $\Gamma$. Condition (1) says that geodesics can have no tall peaks. Suppose $P$ is an edge path with its endpoints in $S(n)$ for some integer $n$ and with another point $z$ outside of $R(n+k)$. A consequence of condition (1) is that such a path is not geodesic.

Condition (2) says that if $x, y \in A[n, n+k]$ and if a geodesic path $P$ joining them is long, then $P$ must leave $A[n, n+k]$. Condition (1) ensures that $P$ dips inside of $R(n)$. Thus, long geodesics tend towards the origin, an expected behavior in negatively curved spaces. In fact, Theorem 2.1 shows that $P$ consists of a segment $P_{0}$ of bounded length containing the innermost points of $P$ and two segments reaching almost directly outward from the endpoints of $P_{0}$. Theorem 2.1 shows these segments are radial quasi-geodesics. Theorem 


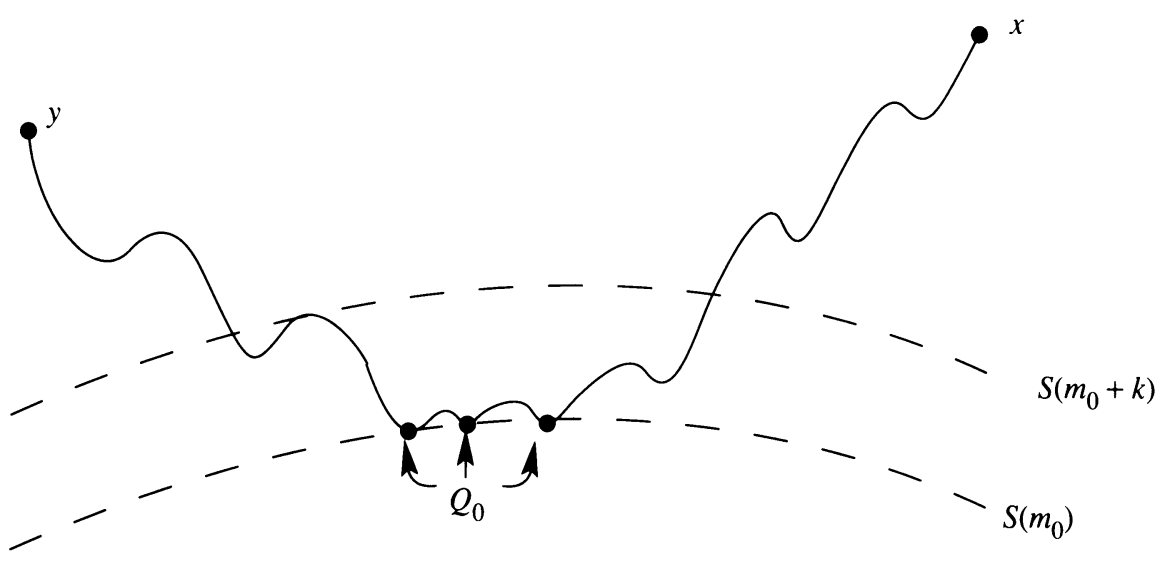

FIGURE 2.1

2.2 uses condition (3) to show that these radial quasi-geodesics are close to the respective sides $[O, x]$ and $[O, y]$. It follows that the triangle $O x y$ is thin.

Theorem 2.1. Suppose conditions (1) and (2) hold in $\Gamma$. Let $O \in \Gamma$ denote a fixed origin. Let $x$ and $y$ be two distinct vertices in $\Gamma$, and let $[x, y]$ denote a geodesic joining them. Then there exist two vertices $x_{0}$ and $y_{0}$ on $[x, y]$ such that

(1) the length of the subsegment $\left[x_{0}, y_{0}\right]$ is bounded by $l-2 k$, and

(2) the segments $\left[x, x_{0}\right]$ and $\left[y_{0}, y\right]$ are radial $(k l, l)$-quasi-geodesics.

Proof. The cases where $x=O$ or $y=O$ are trivial. Assume from now on that $x \neq O$ and $y \neq O$.

Let $Q_{0}$ be the set of innermost vertices in $[x, y]$ and let $m_{0}=d(O, Q)$. If $m_{0}=0$, then the theorem holds with $x_{0}=y_{0}=O$. Now suppose that $m_{0}>0$. I claim that $Q_{0}$ is contained in a connected component of $A\left[m_{0}, m_{0}+k\right] \cap$ $[x, y]$. (See Figure 2.1.) Suppose this is false. Then there are two vertices $a, b \in Q_{0}$ which are in two distinct components of $A\left[m_{0}, m_{0}+k\right] \cap[x, y]$, and there is a point $z \in[x, y]$ which separates $a$ and $b$ for which $d(O, z)>$ $m_{0}+k$. By condition (1) we can shorten $[x, y]$, but this contradicts our hypothesis that $[x, y]$ is geodesic.

Let $P_{0}$ denote the component of $A\left[m_{0}, m_{0}+k\right] \cap[x, y]$ which contains $Q_{0}$. Let $\preceq$ denote the ordering of the points of $[x, y]$ from $y$ to $x$. Let $y_{0}$ and $x_{0}$ be the minimal and maximal vertices of $Q_{0}$. By condition $(2), \ell\left(P_{0}\right) \leq l$. Thus, $\ell\left(\left[x_{0}, y_{0}\right]\right) \leq l-2 k$.

If $x_{0}=x$ and $y_{0}=y$, then we are done with the theorem. Assume then that $x_{0} \neq x$. We will now prove that the segment $\left[x_{0}, x\right]$ is a radial $(k l, l)$-quasigeodesic. If necessary, we can use the same argument to show that $\left[y_{0}, y\right]$ is also a radial $(k l, l)$-quasi-geodesic.

We must consider an arbitrary subedgepath of $\left[x_{0}, x\right]$ bounded by vertices $r_{0}$ and $s$, say, such that $x_{0} \preceq r_{0} \prec s \preceq x$ and $\ell\left(\left[r_{0}, s\right]\right) \geq k l$. We construct a subdivision $\left\{r_{0}, r_{1}, \ldots, r_{n}=s\right\}$ of $\left[r_{0}, s\right]$ as follows.

Let $Q_{1}$ be the set of innermost vertices in $\left[r_{0}, s\right]$ and set $m_{1}=d\left(O, Q_{1}\right)$. We make the following claims:

(i) $m_{0} \leq m_{1}$.

(ii) $m_{1} \leq d\left(O, r_{0}\right) \leq m_{1}+k-2$. 
(iii) $Q_{1} \cup\left\{r_{0}\right\}$ is contained in a connected component of $A\left[m_{1}, m_{1}+k\right] \cap$ $\left[r_{0}, s\right]$.

Statement (i) is clear. The case $m_{0}=m_{1}$ can happen only when $r_{0}=x_{0}$, which implies that $Q_{1}=\left\{r_{0}\right\}$. Statements (ii) and (iii) are then obvious.

Consider the case where $m_{0}<m_{1}$. If (ii) fails, then since $r_{0}$ is a vertex we must have $d\left(O, r_{0}\right) \geq m_{1}+k-1$. Let $a \in Q_{1}$, and let $b$ be a vertex in $\left[x_{0}, r_{0}\right]$ for which $d(O, b)=m_{1}-1$. The current case of item (i) assures us that such a vertex $b$ exists. The segment $[a, b]$, or some subsegment, can be shortened by condition (1), a contradiction.

Now for item (iii). By the same argument used above on $Q_{0}$, all vertices of $Q_{1}$ are in the same component of $A\left[m_{1}, m_{1}+k\right] \cap\left[r_{0}, s\right]$. Suppose therefore that $r_{0}$ is separated from $Q_{1}$ by a point $z$ with $d(O, z)>m_{1}+k$. Then an application of condition (1) allows us to shorten our geodesic segment. Thus, (iii) holds.

Let $P_{1}$ be the component of $A\left[m_{1}, m_{1}+k\right] \cap\left[r_{0}, s\right]$ which contains $r_{0}$ and $Q_{1}$. One endpoint of $P_{1}$ is $r_{0}$. Let $r_{1}$ denote the other endpoint. Then $\ell\left(\left[r_{0}, r_{1}\right]\right) \leq l$ by condition (2). Since $\ell\left(\left[r_{0}, s\right]\right) \geq k l$ and $k \geq 2$ we see that $r_{1} \neq s$. Therefore, $r_{1} \in S\left(m_{1}+k\right)$. (See Figure 2.2.)

We proceed inductively. Having defined $r_{i-1}$ and $P_{i-1}$, we let $Q_{i}$ denote the set of innermost vertices of $\left[r_{i-1}, s\right]$ and set $m_{i}=d\left(O, Q_{i}\right)$. Then, as above, we make the following claims:

(i) $m_{i-1}<m_{i}$

(ii) $m_{i} \leq d\left(O, r_{i-1}\right) \leq m_{i}+k-2$.

(iii) $Q_{i} \cup\left\{r_{i-1}\right\}$ is in a connected component of $A\left[m_{i}, m_{i}+k-1\right] \cap\left[r_{i-1}, s\right]$.

We only need to prove (i). Items (ii) and (iii) follow as above. Suppose $m_{i-1} \geq$ $m_{i}$. Let $b^{\prime} \in Q_{i}$. Let $b \in\left[r_{i-1}, b^{\prime}\right]$ be a vertex such that $d(O, b)=m_{i-1}$. Let $a \in\left[x_{0}, r_{i-1}\right]$ be a vertex such that $d(O, a)=d(O, b)$. Note that $d\left(O, r_{i-1}\right)=$ $m_{i-1}+k$. Thus, some subsegment of $[a, b]$ can be shortened by condition (1).

We define $P_{i}$ and $r_{i}$ in the expected way. As before, $\ell\left(P_{i}\right) \leq l$. If $r_{i} \neq s$, then we note that $r_{i} \in S\left(m_{i}+k\right)$ and we proceed with the induction.

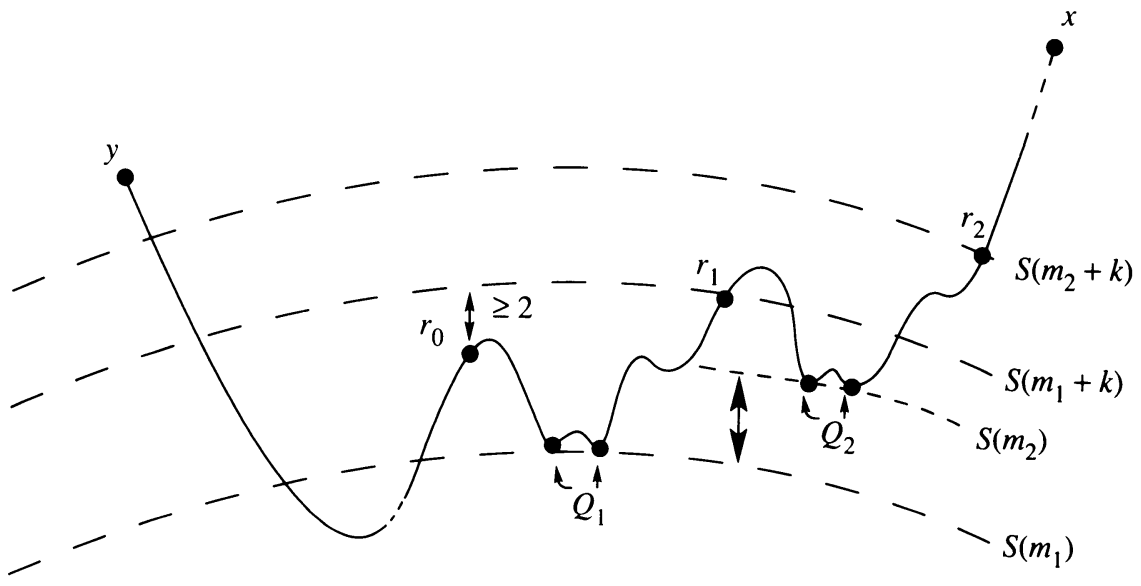

FIGURE 2.2 
Eventually, we arrive at $r_{n}=s$ for some $n \geq k$. For $i=1,2, \ldots, n-1$,

$$
\begin{aligned}
d\left(O, r_{i}\right)-d\left(O, r_{i-1}\right) & =\left(d\left(O, r_{i}\right)-m_{i}\right)-\left(d\left(O, r_{i-1}\right)-m_{i}\right) \\
& \geq k-(k-2) \geq 2
\end{aligned}
$$

and

$$
d\left(O, r_{n}\right)-d\left(O, r_{n-1}\right) \geq-(k-2)
$$

Thus,

$$
\begin{aligned}
d(O, s)-d\left(O, r_{0}\right) & =d\left(O, r_{n}\right)-d\left(O, r_{0}\right)=\sum_{i=1}^{n}\left(d\left(O, r_{i}\right)-d\left(O, r_{i-1}\right)\right) \\
& \geq 2(n-1)-(k-2)=2 n-k \geq n
\end{aligned}
$$

We obtain

$$
\ell\left(\left[r_{0}, s\right]\right) \leq \sum_{i=1}^{n} \ell\left(P_{i}\right) \leq n l \leq l\left|d(O, s)-d\left(O, r_{0}\right)\right|
$$

Theorem 2.2. If $\Gamma$ satisfies the local conditions, then $\Gamma$ has thin triangles $(\delta)$ for some $\delta \geq 0$, and hence the group $G$ is negatively curved.

Proof. Suppose given a geodesic triangle in $\Gamma$. Since $G$ is a group, $\Gamma$ is a homogeneous metric space. We can choose one of the vertices of the triangle to be the identity vertex $O$ and call the other vertices $x$ and $y$.

By Theorem 2.1, there are two vertices $x_{0}, y_{0} \in[x, y]$ such that $\ell\left(\left[x_{0}, y_{0}\right]\right)$ $\leq l-2 k$ and the segments $\left[x_{0}, x\right]$ and $\left[y_{0}, y\right]$ are radial $(k l, l)$-quasigeodesics.

If $d(O, x)-d\left(O, x_{0}\right) \leq 4 k$, then $d\left(x_{0}, x\right) \leq 4 k l$ by Definition 1.2. It follows that each point of $\left[x, x_{0}\right]$ is within $4 k l$ of some point of $[O, x]$.

Suppose on the other hand that $d(O, x)-d\left(O, x_{0}\right) \geq 4 k$. Set $m=d(O, x)$. Order the points of $\left[x_{0}, x\right]$ from $x_{0}$ to $x$. We subdivide $\left[x_{0}, x\right]$ as follows. For $i=1,2, \ldots, n+1$ let $x_{i}$ be the maximal vertex of $\left[x_{0}, x\right] \cap S(m-2 i k)$, where $n+1$ is the largest integer such that $\left[x_{0}, x\right] \cap S(m-2(n+1) k)$ is nonempty. For each $i=1,2, \ldots, n$ let $z_{i}$ be the unique vertex on $[O, x]$ such that $d\left(O, x_{i}\right)=d\left(O, z_{i}\right)$. By condition (3), $d\left(x_{i}, z_{i}\right) \leq 4 k$. Note that $d\left(O, x_{i-1}\right)-d\left(O, x_{i}\right)=2 k$, so by Definition $1.2, d\left(x_{i-1}, x_{i}\right) \leq 2 k l$. Each point of $\left[x_{n}, x\right]$ is within $4 k+k l$ of some point of $[O, x]$. We make a similar subdivision $\left\{y_{1}, \ldots, y_{n^{\prime}}\right\}$ of $\left[y_{0}, y\right]$. Observe that each point of $\left[y_{n^{\prime}}, y\right]$ is within $4 k+k l$ of some point of $[O, y]$. (See Figure 2.3 on the next page.)

We compute a bound on the length of $\left[y_{n^{\prime}}, x_{n}\right]$. Since $d\left(O, x_{n}\right)-d\left(O, x_{0}\right) \leq$ $4 k$, Definition 1.2 implies

$$
\ell\left(\left[x_{0}, x_{n}\right]\right) \leq 4 k l
$$

and similarly

$$
\ell\left(\left[y_{n^{\prime}}, y_{0}\right]\right) \leq 4 k l \text {. }
$$

Recall from the proof of Theorem 3.1 that $\ell\left(\left[x_{0}, y_{0}\right]\right) \leq l-2 k$. The bound we seek is $s=l-2 k+8 k l$ and thus, any point of $[x, y]$ is within $4 k+$ $\max \left\{\frac{1}{2} s, k l\right\}$.

We permute the labels on the vertices and repeat the argument. It follows that $\Gamma$ has thin triangles $(\delta)$ where $\delta=4 k+\max \left\{\frac{1}{2} s, k l\right\}$. 


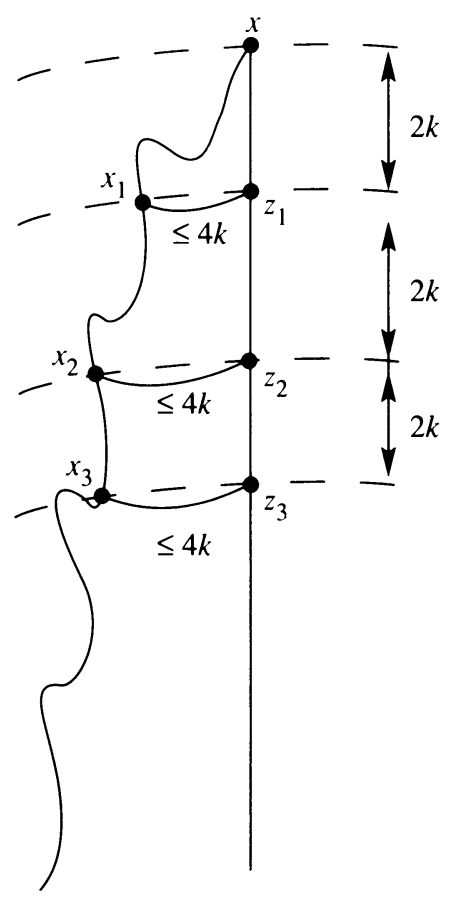

FIGURE 2.3

\section{Negative CURVATURE IMPLIES LOCAL CONDITIONS}

In this section we complete the proof of the equivalence of the local conditions and the thin triangles property.

Theorem 3.1. If $\Gamma$ has thin triangles $(\delta)$ for some $\delta \geq 0$, then $\Gamma$ satisfies the local conditions.

Sketch of proof. We may take $\delta$ to be an integer $\geq 0$. Set $k=\delta+2$ and $l=2 k+4 \delta$. With these values of $k$ and $l$, we prove that each local condition holds. The details of the proof are left to the reader.

\section{AN ALGORITHM FOR DETECTING NEGATIVE CURVATURE}

Suppose we have a finitely presented group $G=\langle A \mid R\rangle$ that we suspect is negatively curved. There is a partial algorithm which will verify this. The algorithm runs as follows.

(1) Set $\delta=0$.

(2) Set $k=2+\delta$ and $l=2 k+4 \delta$.

(3) Set $N=4 k l+4 k$ and enumerate all $N$-types in $\Gamma$.

(4) Check the local conditions in each $N$-type. If they hold, then we are done. If not, increment $\delta$ by 1 and return to step (2).

If $G$ is negatively curved, then this process will eventually terminate; otherwise, it will run forever. This is the best kind of algorithm we can hope for since negative curvature is a Markov property [LS, p. 192]

The only step not discussed so far is the enumeration of $N$-types. This is made reasonably easy by the fact that negatively curved groups are automatic 
[ECH, Sec. 3.4]. In fact, they are shortlex automatic and this fact is independent of the presentation. Furthermore, computer programs exist which can explicitly compute the shortlex automatic structure [EHR]. This structure makes it possible to efficiently build segments of the Cayley graph. It also enables us to find a number $R$ whose value is independent of $N$ such that all $N$-types can be found in the ball of radius $R+2 N$. See [S]. We construct this ball and enumerate all $N$-types we find in it.

If $G$ is not negatively curved, then it may or may not have a shortlex automatic structure. If not, the programs designed to find it will never terminate. If a shortlex automatic structure is found, we can then proceed with the algorithm above, but it will never terminate.

Algorithms for detecting negative curvature are also discussed by Papasoglu $[\mathrm{P}]$ and Gromov [Gr, 2.3F].

\section{REFERENCES}

[ABC] J. M. Alonso, T. Brady, D. Cooper, V. Ferlini, M. Lustig, M. Mihalik, M. Shapiro, and H. Short, Notes on hyperbolic groups, Group Theory from a Geometric Viewpoint (E. Ghys, A. Haefliger, H. Short, and A. Verjovsky, eds.), World Scientific, Singapore, 1991.

[B] B. H. Bowditch, Notes on Gromov's hyperbolicity criterion for path-metric spaces, Group Theory from a Geometric Viewpoint (E. Ghys, A. Haefliger, and A. Verjovsky, eds.), World Scientific, Singapore, 1991.

[GdlH] W. E. Ghys and P. de la Harpe (eds.), Sur les groupes hyperboliques d'après Mikhael Gromov, Progr. Math., Birkhaüser, Zürich, 1990.

[C] J. Cannon, The theory of negatively curved spaces and groups, Ergodic Theory, Symbolic Dynamics, and Hyperbolic Spaces (Tim Bedford, Michael Keane, and Caroline Series, eds.), Oxford Univ. Press, Oxford, New York and Tokyo, 1991, pp. 315-369.

[ECH] D. Epstein, J. Cannon, D. Holt, S. Levy, M. Paterson, and W. Thurston, Word processing in groups, A. K. Peters, 1992.

[EHR] D. Epstein, D. Holt, and S. Rees, The use of Knuth-Bendix methods to solve the word problem in automatic groups, J. Symbolic Comput. 12 (1991), 397-414.

[Ge] S. M. Gersten, Isoperimetric and isodiametric functions of finite presentations, preprint, University of Utah.

[Gh] E. Ghys, Les groupes hyperboliques, Sém. Bourbaki, Exposé no. 722, Astérisque 1989/90 (1990), 203-238.

[Gr] M. Gromov, Hyperbolic groups, Essays on Group Theory (S. M. Gersten, ed.), Math. Sci. Res. Inst. Publ., vol. 8, Springer-Verlag, New York, 1987, pp. 75-263.

[LS] R. C. Lyndon and P. E. Schupp, Combinatorial group theory, Springer-Verlag, New York, 1977.

[P] P. Papasoglu, Detecting hyperbolicity, preprint, Columbia University.

[S] Paul Shawcroft, Identifying local structures in certain automatic groups, preprint, Brigham Young Univ.

Department of Mathematics, Brigham Young University, Provo, Utah 84602

E-mail address: paul@math.byu.edu 